\title{
Association of variable number of tandem repeat polymorphism in the $I L-4$ gene with ischemic stroke in the Chinese Uyghur population
}

\author{
Y.-Q. Tong1,2,3*, J.-J. Ye ${ }^{2 *}$, Z.-H. Wang1*, Y.-W. Zhang ${ }^{3 *}$, F.-X. Zhan ${ }^{2}$,

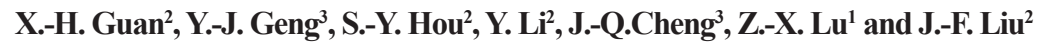 \\ ${ }^{1}$ School of Public Health, Tongji Medical College, \\ Huazhong University of Science and Technology, Wuhan, China \\ ${ }^{2}$ Hubei Center for Disease Control and Prevention, Wuhan, China \\ ${ }^{3}$ Shenzhen Center for Disease Control and Prevention, Shenzhen, China \\ *These authors contributed equally to this study. \\ Corresponding authors: J.-F. Liu / Z.-X. Lu \\ E-mail: L_jiafa@163.com / luzuxun@126.com
}

Genet. Mol. Res. 12 (3): 2423-2431 (2013)

Received April 10, 2012

Accepted March 16, 2013

Published May 13, 2013

DOI http://dx.doi.org/10.4238/2013.May.13.2

\begin{abstract}
Ischemic stroke (IS) is a multifactorial disorder, and genetic factors act as important contributors to its onset and progression. Inflammation is a key event that is closely associated with the pathophysiology of IS. The association of genetic polymorphisms of inflammatory cytokines with IS remains poorly understood. We investigated the relationship between the variable number of tandem repeats (VNTR) for IL-4, which is an important biomarker of inflammation, and the risk of IS. To assess the nature of the VNTR polymorphism in $I L-4$ and identify any links with IS, we recruited 200 subjects from a unique population that has $60 \%$ European and $40 \%$ East Asian ancestry. The subjects comprised 100 IS patients diagnosed using magnetic resonance imaging within $24 \mathrm{~h}$ of symptom onset and 100 age-, gender- and ethnicity-matched normal healthy controls. VNTR was identified using high-performance capillary electrophoresis with
\end{abstract}


specially designed tailed primers. The $I L-4$ VNTR polymorphism was significantly associated with IS after adjustment for cardiovascular risk factors $(\mathrm{OR}=0.571,95 \% \mathrm{CI}=0.330-0.949, \mathrm{P}<0.05)$. Our data indicate that $I L-4$ VNTR polymorphism may affect susceptibility to IS in the Chinese Uyghur population. Moreover, total cholesterol, fasting blood glucose, waist-to-hip ratio, hypertension, history of heart diseases, and negative events may increase the risk of IS, with a trend for HDL to be a protective factor for IS in the Uyghur population.

Key words: High-performance capillary electrophoresis; IL-4; Variable number of tandem repeats; Ischemic stroke; Uyghur population

\section{INTRODUCTION}

Ischemic stroke (IS) is a common neurological disease with a variety of causes and is manifested as atherosclerosis or gradual cholesterol deposition. Chronic low-grade inflammation and activation of the innate immune system are closely involved in the pathogenesis of IS (Weyrich et al., 2009). Mounting evidence from in vivo and ex vivo studies showed that inflammatory cytokines play a crucial role in the development of IS (Castillo et al., 2009; Kashyap et al., 2009; Kriz and Lalancette-Hébert, 2009). Cross-sectional studies have provided support for the hypothesis that chronic subclinical inflammation may be associated with atherosclerosis and may precede the progression of clinically overt IS (Kang et al., 2009).

Interleukin-4 (IL-4) is an important anti-inflammatory cytokine characterized by the elaboration of other anti-inflammatory molecules, such as IL-10 and -1RN. IL-4 directs macrophages towards a phenotype that regulates humoral immunity and Ig production (Romagnani, 1995; Miossec and van den Berg, 1997). IL-4 also shifts the inflammatory balance by inhibiting the secretion of the pro-inflammatory cytokines IL- $1 \beta$, TNF- $\alpha$, and IL- 6 from macrophages. Disturbance of the anti-inflammatory response might be a critical component of the chronic inflammation found in IS (Um and Kim, 2009). The $I L-4$ gene contains a 70-bp variable number of tandem repeat (VNTR) polymorphism in intron 3 that is associated with IL-4 production. It was proposed that an increased responsiveness of the $2 \mathrm{R}$ allele to transcriptional activation might lead to the overexpression of $I L-4$ (Vandenbroeck et al., 1997). Furthermore, patients presented elevated IL-4 levels during the acute stage of cerebral infarction (Kim et al., 2000). The VNTR polymorphism of the $I L-4$ gene has been widely shown to be associated with a number of inflammatory diseases, including multiple sclerosis and idiopathic thrombocytopenic purpura as well as cerebrovascular disease (Vandenbroeck et al., 1997; Chen et al., 2007; Um and Kim, 2009). In addition, several in vitro studies showed that IL-4 regulates the JAK and STAT signal transduction pathways, consequently playing an important role in atherosclerosis and various inflammatory diseases (Saggini et al., 2011; Bao et al., 2012). Some researchers have even proposed that IL-4 has possible therapeutic potential in the treatment of inflammation-related disorders (Kleemann et al., 2008; Profumo et al., 2008). Because of the biological plausibility for its involvement in atherosclerosis, its association with inflammatory diseases, and its potential influence on IL-4 and other cytokine production (Lee et al., 2010), we investigated IL-4 VNTR polymorphism in IS patients.

The Uyghur population is a minority group that originated from Turkish nomads liv- 
ing in Xinjiang, and it has its own language, religious beliefs, and lifestyles that may be very different from either the Han population or American/European populations. The Uyghur represent a unique population, with a typical admixture of Eastern and Western anthropometric traits in China. A recent study confirmed that the Uyghur population has 60\% European and $40 \%$ East Asian ancestry (Xu et al., 2008).

The nature of association of IL-4 VNTR with Chinese Uyghur IS patients remains unknown. Hence, as a first step, it is important to evaluate whether the VNTR of these cytokines have a functional influence on the susceptibility of the Uyghur population to IS. Therefore, we evaluated the association of IL-4 VNTR with IS in a population-based study among the Chinese Uyghur population.

\section{MATERIAL AND METHODS}

\section{Patients and clinical evaluation}

The current study protocol was performed from September 2005 to February 2009, with prior approval from the Local Ethics Committee and parents of the inpatients attending the stroke unit of 2 large general hospitals in Xinjiang. Because of religious beliefs and the harsh medical and living environment in which this ethnic group is clustered, the collection of blood sample was extremely difficult. All subjects provided informed written consent. The diagnosis of IS was established according to the international classification of the World Health Organization (Wood, 1980). The stroke subtypes were evaluated according to the Oxfordshire classification (Bamford et al., 1990, 1991). Following screening with head computed tomography or magnetic resonance imaging tests, a total of 100 subjects who were clinically diagnosed with lacunar infarction within $24 \mathrm{~h}$ of presenting the onset of symptoms were enrolled in the current study. Furthermore, control subjects, residing in the same communities as the cases, were determined to be free of IS and related neurological diseases on the basis of medical history, clinical examinations, and electroencephalogram. The subjects were matched for age (range, 5 years), gender, ethnic origin, and residence. Control subjects who reported a history of cardiovascular events other than stroke were eligible. The patients and controls were interviewed using a structured questionnaire by trained interviewers. Besides a neurological and family history of hypertension and diabetes mellitus, the following vascular risk factors and environmental factors were recorded: history of alcohol drinking, cigarette smoking, tea drinking, physical exercise, and body mass index (BMI). Waist-to-hip ratios (WHR) were measured over 1 layer of clothing by using a fiberglass tape. Hip circumference was measured by placing the tape measure at the level of the great trochanter or approximately 4 inches below the waistline. WHR was calculated by dividing waist circumference by hip circumference. Negative events included 10 usual kinds of negative life events such as death of significant others, severe illness of self or significant others, negative socioeconomic circumstances and so on.

\section{Sample processing and genotyping}

After obtaining informed consent from both the groups, a 5-mL blood sample was taken in ethylenediaminetetraacetic acid and plain vials. Genomic DNA was extracted from 
a peripheral blood leukocyte pellet by using the standard salting-out method with a DNA extraction kit (Axygen, Union City, CA, USA). DNA samples were stored at $-80^{\circ} \mathrm{C}$ before use.

\section{IL-4 intron-3 VNTR genotyping by molecular genetic analyses}

The region that contains the VNTR of 70 bp within the IL-4 intron-3 was amplified using polymerase chain reaction (PCR). Amplification was performed with 3 primers: the forward primer with an M13 universal tail, a reverse primer, and a labeled primer (with a Beckman-Coulter dye-cy5). The reactions were performed using a total volume of $10 \mu \mathrm{L}$. This volume contained $100 \mathrm{ng}$ genomic DNA, $10 \mathrm{pmol}$ of each primer, $1 \mathrm{X}$ Taq polymerase buffer, and $0.5 \mathrm{U}$ Thermo-Start ${ }^{\mathrm{TM}}$ DNA polymerase (Thermo-Start ${ }^{\mathrm{TM}}$ Master Mix, AB gene, UK). The final products contained a fluorescence dye detected using the Beckman Coulter Genetic Analysis System. Detailed information about the PCR conditions and primer sequences are summarized in Table 1. Alleles of 2 cytokines were analyzed using high-performance capillary electrophoresis with GenomeLab Separation Gel (Beckman Coulter, USA) and a DNA size standard kit-600 (Beckman Coulter) on the CEQ 8800 Genetic Analysis System.

Table 1. Tailed primers, PCR conditions, and genotyping.
\begin{tabular}{llc}
\hline Gene & Primer sequence (5'-3') & Ta/extension time in s/cycles \\
\hline IL-4 & $\begin{array}{l}\text { CACGACGTTGTAAAACGACTAGGCTGAAAGGGGGAAAGC (F) } \\
\text { CTGTTCACCTCAACTGCTCC (R) } \\
\text { Cy5-CACGACGTTGTAAAACGAC (M-13) }\end{array}$ & $60 / 60 / 40$ \\
\hline
\end{tabular}

$\mathrm{Ta}=$ annealing temperature $\left({ }^{\circ} \mathrm{C}\right) ; \mathrm{F}=$ forward; $\mathrm{R}=$ reverse.

To improve genotyping quality and validation, quality control was performed on the entire batch of 200 samples. Every plate had negative controls, and the call rate for negative controls was $100 \% ; 10 \%$ of samples were re-genotyped, and 10 randomly selected samples were re-sequenced. The results were found to be reproducible with no observed discrepancy in genotyping.

\section{Determination of biological variables}

Fasting blood glucose, total cholesterol (TC), triglyceride, HDL, and LDL were assayed using standard laboratory procedures in the Department of the Clinical Laboratory at the local hospital. Diabetes mellitus was diagnosed when the subject had a fasting glucose level $>7.8 \mathrm{mM}$ or a glucose level $>1.1 \mathrm{mM}$ at $2 \mathrm{~h}$ after an oral glucose challenge, or both.

\section{Statistical analysis}

Allele frequencies, genotype frequencies, and carriage rates of the alleles in all the groups were compared using the Fisher exact test with the SPSS software program (version 12.0, SPSS Inc., Chicago, IL, USA). Data on quantitative characteristics were reported as means \pm standard deviation. Comparisons between groups were performed using the $\chi^{2}$ test (nominal data) or the Student $t$-test (interval data). Allele frequency was calculated as the number of occurrences of the test allele in the population divided by the total number 
of alleles. The carriage rate was calculated as the number of individuals carrying at least 1 copy of the test allele divided by the total number of individuals. All $\mathrm{P}$ values were two-sided, and differences were considered statistically significant when $\mathrm{P}<0.05$. The Hardy-Weinberg equilibrium was tested using the $\chi^{2}$ method. The odds ratio (OR) at a $95 \%$ confidence interval $(95 \% \mathrm{CI})$ was determined to describe the strength of association using a logistic regression model.

\section{RESULTS}

In total, 100 IS patients (mean age: $64.12 \pm 9.36$ years) and age-, gender- and ethnicity-matched healthy controls (mean age: $63.18 \pm 10.19$ years) were evaluated for $I L-4$ gene VNTR polymorphisms. The results indicate that WHR, hypertension, and a history of heart diseases may increase the risk of IS. However, there was a trend for HDL to be a protective factor for IS. triglyceride, TC, LDL, and other parameters are listed in Table 2.

Table 2. Demographic characteristics and distribution of traditional risk factors in Chinese Uyghur population.

\begin{tabular}{|c|c|c|c|}
\hline \multirow[t]{2}{*}{ Characteristic } & \multicolumn{2}{|c|}{ Uyghur } & \multirow[t]{2}{*}{$\mathrm{P}^{\mathrm{c}}$} \\
\hline & Cases $(\mathrm{N}=100)$ & Controls $(\mathrm{N}=100)$ & \\
\hline Gender (M/F) & $58 / 42$ & $58 / 42$ & \\
\hline Age (years) & $64.12 \pm 9.36$ & $63.18 \pm 10.19$ & 0.498 \\
\hline BMI $\left(\mathrm{kg} / \mathrm{m}^{2}\right)$ & $24.51 \pm 3.47$ & $23.56 \pm 2.73$ & 0.040 \\
\hline WHR & $0.89 \pm 0.07$ & $0.80 \pm 0.06$ & 0.000 \\
\hline FH of hypertension & $36.00 \%$ & $46.00 \%$ & 0.151 \\
\hline FH of diabetes & $15.00 \%$ & $25.00 \%$ & 0.077 \\
\hline History of hypertension ${ }^{\mathrm{a}}$ & $59.00 \%$ & $11.00 \%$ & 0.000 \\
\hline History of diabetes & $10.00 \%$ & $8.00 \%$ & 0.621 \\
\hline History of heart diseases & $8.00 \%$ & $0.00 \%$ & 0.007 \\
\hline Smoking ${ }^{\mathrm{b}}$ & $17.00 \%$ & $19.00 \%$ & 0.713 \\
\hline Current alcohol drinker & $16.00 \%$ & $26.00 \%$ & 0.083 \\
\hline Negative events & $14.00 \%$ & $1.00 \%$ & 0.000 \\
\hline Tea consumption & $98.00 \%$ & $100.00 \%$ & 0.155 \\
\hline Triglyceride (mM) & $1.69(1.07)$ & $1.66(0.92)$ & 0.849 \\
\hline Cholesterol (mM) & $4.53(1.24)$ & $5.65(1.23)$ & 0.000 \\
\hline $\operatorname{HDL}(\mathrm{mM})$ & $1.06(0.37)$ & $1.39(0.38)$ & 0.000 \\
\hline LDL (mM) & $2.70(0.77)$ & $2.78(0.53)$ & 0.767 \\
\hline FBG $(\mathrm{mM})$ & $6.51(2.16)$ & $5.35(1.71)$ & 0.000 \\
\hline \multicolumn{4}{|c|}{ 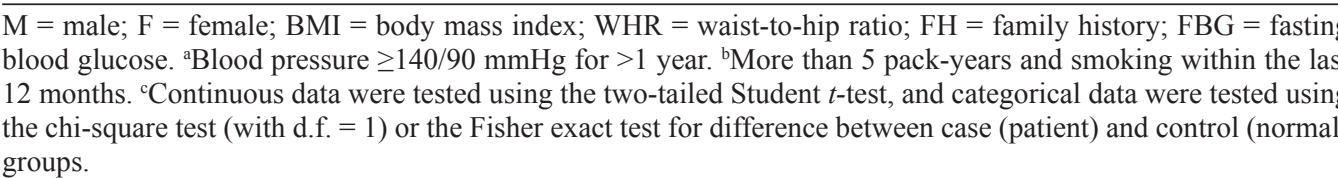 } \\
\hline
\end{tabular}

Three main genotypes of IL-4 were identified in the cases and controls, as shown in Figure 1A-C. The 2R/2R genotype is the most common genotype in the Chinese Uyghur population. The comparative distribution of genotype in patients versus controls showed that the $I L-4$ gene polymorphism might be a risk factor for IS in a dominant model $(\mathrm{OR}=0.515$, $95 \% \mathrm{CI}=0.280-0.949)$. Regarding allele frequency and carriage rate, there was also a significant association $(\mathrm{P}<0.05)$ among patients and controls $(\mathrm{OR}=0.554,95 \% \mathrm{CI}=0.322-0.952)$ (Table 3). Moreover, after adjustment for hypertension, diabetes, smoking, alcohol drinking, BMI, and WHR, there was still a significant difference in the carriage rate of $I L-4$ VNTR polymorphism when assuming a dominant and additive model of inheritance $(\mathrm{P}<0.05)$ (Table 4). 

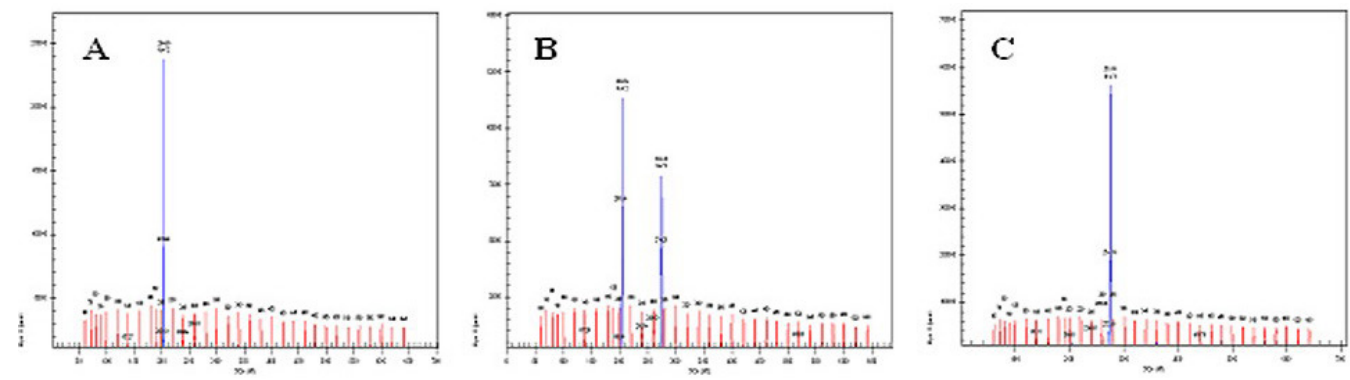

Figure 1. A. Homozygous $(2 \mathrm{R} / 2 \mathrm{R})$ of $I L-4$ variable number of tandem repeats (VNTR). B. Heterozygous (2R/3R) of $I L-4$ VNTR. C. Homozygous (3R/3R) of $I L-4$ VNTR.

Table 3. $I L-4$ VNTR genotype and allele distributions between cases and controls.

\begin{tabular}{lcccc}
\hline Genotype & IS & Controls & OR $(95 \% \mathrm{CI})$ & $\mathrm{P}$ \\
\hline IL-4 VNTR & & & & \\
2R/2R & 76 & 62 & $0.536(0.287-1.000)$ & $<0.05$ \\
2R/3R & 23 & 35 & $0.272(0.028-2.680)$ & $<0.05$ \\
3R/3R & 1 & 3 & & \\
2R/3R+3R/3R & 24 & 38 & $0.515(0.280-0.949)$ & $<0.05$ \\
Allele & 175 & 159 & & \\
Allele2R & 25 & 41 & & \\
Allele3R & $12.50 \%$ & $20.50 \%$ & & \\
MAF & & & \\
\hline MAF = minor allele frequency; IS = ischemic stroke; OR = odds ratio; $95 \% \mathrm{CI}=95 \%$ confidence interval.
\end{tabular}

$\mathrm{MAF}=$ minor allele frequency; $\mathrm{IS}=$ ischemic stroke; $\mathrm{OR}=$ odds ratio $95 \% \mathrm{CI}=95 \%$ confidence interval.

$\begin{aligned} & \text { Table 4. Association of } I L-4 \text { variable number of tandem repeat (VNTR) polymorphisms in the risk of ischemic } \\
& \text { stroke among the Uyghur population. }\end{aligned}$
\begin{tabular}{lcc} 
& Adjusted OR $(95 \% \mathrm{CI})$ & $\mathrm{P}$ \\
\hline$I L-4$ VNTR & & 0.03 \\
Dominant & $0.557(0.321-0.950)$ & 0.31 \\
Recessive & $0.337(0.041-3.195)$ & 0.03 \\
Additive & $0.571(0.330-0.949)$ &
\end{tabular}

Multivariate logistic regression analysis after adjustment for hypertension, diabetes, smoking, alcohol drinking, body mass index, waist-to-hip ratio. $\mathrm{OR}=$ odds ratio; $95 \% \mathrm{CI}=95 \%$ confidence interval.

\section{DISCUSSION}

Previous studies have reported that acute infections, accompanied by acute-phase inflammation reactions and a prothrombotic state, are associated with an increased risk of acute vascular events, such as myocardial infarction and IS (Syrjänen et al., 1988; Lindsberg and Grau, 2003; Zeller et al., 2005). Susceptibility to infection/inflammation and/or risk of atherosclerosis and IS events were shown to be associated with genetic variations in genes and cytokine receptor genes (Worrall et al., 2003; Markus et al., 2006; Steinbrugger et al., 2009). However, data relating these inflammatory cytokine polymorphisms to cerebrovascular events are absent or debatable (Lalouschek et al., 2006; Tso et al., 2007; Yamada et al., 2008). 
Many previous studies have demonstrated that elevated IL-4 production could indicate inflammatory diseases (Saggini et al., 2011). In particular, there is little questions that the elevated production of IL-4 serves as an excellent marker of IS in vitro (Kim et al., 2000). However, the role of the $I L-4$ VNTR polymorphism in the risk for IS remains unclear. Previous studies found a unique association of the $I L-4$ VNTR polymorphism with the rupture of unstable atherosclerotic plaque, which is the pathological substrate for acute ischemic events (Peeters et al., 2009; Park et al., 2011). In addition, the $I L-4$ VNTR polymorphism has been reported to affect transcriptional activity, inducing high expression of $I L-4$, which leads to a shift in the Th1/Th2 balance toward Th2 (Vandenbroeck et al., 1997). Through these possible mechanisms, the $I L-4$ VNTR polymorphism may play a critical role in inflammation that is related to the pathogenesis of IS. Um and Kim (2009) demonstrated a significant association of the $I L-4$ VNTR polymorphism with cerebral infarction in a Korean population. In patients with IS, a higher prevalence of the two-repeat allele $(\mathrm{IL}-4 * 2)$ was reported in Koreans, but whether the $I L-4$ VNTR polymorphism is a risk factor in Chinese Uyghur IS patients remains largely unknown. The data from the Chinese Uyghur participants in the current study showed that the 3R allele of the VNTR polymorphism appears to be a decreased risk for IS $(\mathrm{OR}=0.571,95 \% \mathrm{CI}=0.330-0.949)$, after adjustment for hypertension, diabetes, smoking, alcohol drinking, BMI, and WHR $(\mathrm{P}<0.05)$. The findings of the current study confirmed and developed the previous results obtained for Koreans. These interesting findings suggest that the $I L-4$ VNTR polymorphism may have wider genetic inferences of locus effects on the risk of IS across different races with different geographic origins and lifestyles.

The current study had certain strengths, including a relatively unique population; the use of rigorous methods to diagnose IS, including a formal assessment of the reliability of interpretations of head $\mathrm{CT}$ or/and magnetic resonance imaging findings; and a biologically plausible a priori rationale for selecting the candidate genes. Moreover, the Uyghur population is a unique ethnic group, with a distinctive genetic background. Finally, we pioneered the use of a rapid protocol that was more sensitive and precise than traditional PCR and agarose gel electrophoresis to identify the VNTR of $I L-4$ at a level of accuracy of $1 \mathrm{bp}$. However, our study also had some limitations. First, it was a case-control study, and selection bias could not be completely excluded for the group of patients with IS. Second, because of the long timespan of our study design, the preservation and measurement of IL-4 levels in serum as well as the association studies of the polymorphism could not be performed. Third, our findings might apply only to the Chinese Uyghur population. Finally, the sample-size of our selected population was relatively small and might not be representative of the population as a whole.

Overall, we demonstrated that TC, fasting blood glucose, WHR, hypertension, history of heart disease, and negative events may increase the risk of IS, with a trend for HDL as a protective factor for IS in the Uyghur population. Moreover, the IL-4 VNTR polymorphism may be an independent protective contributor for IS in the Chinese Uyghur population. Further exploration with a larger, more ethnically diverse population is required to improve our understanding about the association of $I L-4$ genetic polymorphism with IS and to precisely elucidate the complicated pathophysiological mechanisms.

\section{ACKNOWLEDGMENTS}

Research supported by the National Natural Science Foundation of China (NSFC $\# 81172153$ ) and a grant (\#2008CDB192) from the Hubei Province. We are grateful to all the 
participants of this study and thank Yi-Jie Geng and Dong-Feng Gu for their technical assistance as well as David Cushley for language assistance.

\section{REFERENCES}

Bamford J, Dennis M, Sandercock P, Burn J, et al. (1990). The frequency, causes and timing of death within 30 days of a first stroke: the Oxfordshire Community Stroke Project. J. Neurol. Neurosurg. Psychiatry 53: 824-829.

Bamford J, Sandercock P, Dennis M, Burn J, et al. (1991). Classification and natural history of clinically identifiable subtypes of cerebral infarction. Lancet 337: 1521-1526.

Bao L, Shi VY and Chan LS (2012). IL-4 regulates chemokine CCL26 in keratinocytes through the Jak1, 2/Stat6 signal transduction pathway: Implication for atopic dermatitis. Mol. Immunol. 50: 91-97.

Castillo J, Alvarez-Sabin J, Martinez-Vila E, Montaner J, et al. (2009). Inflammation markers and prediction of post-stroke vascular disease recurrence: the MITICO study. J. Neurol. 256: 217-224.

Chen X, Xu J, Chen Z, Zhou Z, et al. (2007). Interferon-gamma +874A/T and interleukin-4 intron3 VNTR gene polymorphisms in Chinese patients with idiopathic thrombocytopenic purpura. Eur. J. Haematol. 79: 191-197.

Kang DW, Yoo SH, Chun S, Kwon KY, et al. (2009). Inflammatory and hemostatic biomarkers associated with early recurrent ischemic lesions in acute ischemic stroke. Stroke 40: 1653-1658.

Kashyap RS, Nayak AR, Deshpande PS, Kabra D, et al. (2009). Inter-alpha-trypsin inhibitor heavy chain 4 is a novel marker of acute ischemic stroke. Clin. Chim. Acta 402: 160-163.

Kim HM, Shin HY, Jeong HJ, An HJ, et al. (2000). Reduced IL-2 but elevated IL-4, IL-6, and IgE serum levels in patients with cerebral infarction during the acute stage. J. Mol. Neurosci. 14: 191-196.

Kleemann R, Zadelaar S and Kooistra T (2008). Cytokines and atherosclerosis: a comprehensive review of studies in mice. Cardiovasc. Res. 79: 360-376.

Kriz J and Lalancette-Hébert M (2009). Inflammation, plasticity and real-time imaging after cerebral ischemia. Acta Neuropathol. 117: 497-509.

Lalouschek W, Schillinger M, Hsieh K, Endler G, et al. (2006). Polymorphisms of the inflammatory system and risk of ischemic cerebrovascular events. Clin. Chem. Lab. Med. 44: 918-923.

Lee YW, Kim PH, Lee WH and Hirani AA (2010). Interleukin-4, Oxidative Stress, Vascular Inflammation and Atherosclerosis. Biomol. Ther. 18: 135-144.

Lindsberg PJ and Grau AJ (2003). Inflammation and infections as risk factors for ischemic stroke. Stroke 34: 2518-2532.

Markus HS, Labrum R, Bevan S, Reindl M, et al. (2006). Genetic and acquired inflammatory conditions are synergistically associated with early carotid atherosclerosis. Stroke 37: 2253-2259.

Miossec P and van den Berg W (1997). Th1/Th2 cytokine balance in arthritis. Arthritis Rheum. 40: 2105-2115.

Park HJ, Kim MJ, Kang SW, Kim SK, et al. (2011). Association between interleukin-4 gene polymorphisms and intracerebral haemorrhage in Korean population. Int. J. Immunogenet. 38: 321-325.

Peeters W, Hellings WE, de Kleijn DP, de Vries JP, et al. (2009). Carotid atherosclerotic plaques stabilize after stroke: insights into the natural process of atherosclerotic plaque stabilization. Arterioscler. Thromb. Vasc. Biol. 29: 128-133.

Profumo E, Buttari B, Tosti ME, Siracusano A, et al. (2008). Association of intracellular pro- and anti-inflammatory cytokines in peripheral blood with the clinical or ultrasound indications for carotid endarterectomy in patients with carotid atherosclerosis. Clin. Exp. Immunol. 152: 120-126.

Romagnani S (1995). Biology of human TH1 and TH2 cells. J. Clin. Immunol. 15: 121-129.

Saggini A, Anogeianaki A, Maccauro G, Tete S, et al. (2011). Cholesterol, cytokines and diseases. Int. J. Immunopathol. Pharmacol. 24: 567-581.

Steinbrugger I, Haas A, Maier R, Renner W, et al. (2009). Analysis of inflammation- and atherosclerosis-related gene polymorphisms in branch retinal vein occlusion. Mol. Vis. 15: 609-618.

Syrjänen J, Valtonen VV, Iivanainen M, Kaste M, et al. (1988). Preceding infection as an important risk factor for ischaemic brain infarction in young and middle aged patients. Br. Med. J. 296: 1156-1160.

Tso AR, Merino JG and Warach S (2007). Interleukin-6 174G/C polymorphism and ischemic stroke: a systematic review. Stroke 38: 3070-3075.

Um JY and Kim HM (2009). Polymorphisms of RANTES and IL-4 genes in cerebral infarction. J. Mol. Neurosci. 37: 1-5.

Vandenbroeck K, Martino G, Marrosu M, Consiglio A, et al. (1997). Occurrence and clinical relevance of an interleukin-4 gene polymorphism in patients with multiple sclerosis. J. Neuroimmunol. 76: 189-192.

Weyrich AS, Skalabrin EJ and Kraiss LW (2009). Targeting the inflammatory response in secondary stroke prevention: a role for combining aspirin and extended-release dipyridamole. Am. J. Ther. 16: 164-170. 
Wood PH (1980). Appreciating the consequences of disease: the international classification of impairments, disabilities, and handicaps. WHO Chron. 34: 376-380.

Worrall BB, Azhar S, Nyquist PA, Ackerman RH, et al. (2003). Interleukin-1 receptor antagonist gene polymorphisms in carotid atherosclerosis. Stroke 34: 790-793.

Xu S, Huang W, Qian J and Jin L (2008). Analysis of genomic admixture in Uyghur and its implication in mapping strategy. Am. J. Hum. Genet. 82: 883-894.

Yamada Y, Ichihara S and Nishida T (2008). Proinflammatory gene polymorphisms and ischemic stroke. Curr. Pharm. Des. 14: 3590-3600.

Zeller JA, Lenz A, Eschenfelder CC, Zunker P, et al. (2005). Platelet-leukocyte interaction and platelet activation in acute stroke with and without preceding infection. Arterioscler. Thromb. Vasc. Biol. 25: 1519-1523. 